\title{
Accuracy of registrations between cone-beam computed tomography and conventional computed tomography images and dose mapping methods in RaySearch software for the bladder during brachytherapy of cervical cancer patients
}

\author{
Paweł Czajkowski, MScl, Grzegorz Zwierzchowski, PhD2,3, Tomasz Piotrowski, PhD, DSc 2,3 \\ 'Department of Medical Physics, Gdynia Oncology Centre, Gdynia, Poland, ${ }^{2}$ Department of Electroradiology, Poznan University of Medical \\ Sciences, Poznan, Poland, ${ }^{3}$ Department of Medical Physics, Greater Poland Cancer Centre, Poznan, Poland
}

\begin{abstract}
Purpose: The aim of the study was to assess selected methods of image registration available in the RaySearch software and their impact on the accuracy of mapping of doses deposited in the bladder during brachytherapy (BRT) of cervical cancer in images used during external beam radiotherapy (EBRT).

Material and methods: The study was based on data from ten patients. Cone-beam computed tomography (CBCT) images (BRT) were aligned with CT images (EBRT) using four registration methods: Reg_1 (rigid), Reg_2a, Reg_2b (hybrid), and Reg_3 (biomechanical). Image mapping accuracy was evaluated based on bladder's anatomy. Sørensen-Dice coefficient (DSC) values were analyzed for all the registrations. Discrepancies between triangular mesh points set on the basis of bladder contours were analyzed. Dose distributions from BRT were transformed according to registration results and mapped on CT images. Original BRT doses deposited in $2 \mathrm{~cm}^{3}$ volume of the bladder were compared to those transformed and associated with bladder's volume determined on CT images.

Results: Mean DSC values amounted to 0.36 (Reg_1), 0.87 and 0.88 (Reg_2a and Reg_2b), and 0.97 (Reg_3). Significant differences were found between DSC for the following comparisons: Reg_3/Reg_1 $(p=0.001)$, Reg_2a/Reg_1 $(p=0.011)$, and Reg_2b/Reg_1 $(p=0.014)$. The lowest discrepancies between triangular mesh points were for Reg_3 $(p<0.001$, Reg_3 vs. Reg_1, and $p=0.039$, Reg_3 vs. Reg_2b). Finally, the lowest discrepancies between the original and transformed doses were found for Reg_3. Nevertheless, only 5 out of 10 observations for Reg_3 yielded error of less than $5 \%$.

Conclusions: Biomechanical registration (Reg_3) enabled the most accurate alignment between CBCT and CT images. Satisfactory registration results of anatomical structures do not guarantee a correct mapping of primary BRT doses on the bladder delineated on CT images during EBRT. The results of dose transformation based on biomechanical registration had an error of less than $5 \%$ for only $50 \%$ of the observations.
\end{abstract}

J Contemp Brachytherapy 2020; 12, 6: 593-600 DOI: https://doi.org/10.5114/jcb.2020.101693

Key words: deformable image registration, dose mapping, brachytherapy, cervix.

\section{Purpose}

Radiotherapy of cervical cancer is usually delivered as a combined treatment [1]. Dose deposited in the target volume is delivered through a system of several external photon radiation beams (EBRT) and intracavitary application of ionizing radiation sources (BRT). EBRT is a multifractional radiation, with total dose (e.g., 50.4 Gy) delivered in more than ten (in this case 28 ) radiation fractions [2]. In BRT, total dose (e.g., $28 \mathrm{~Gy}$ ) is delivered in several (in this case 4) radiation fractions [3]. The combination of EBRT and BRT enables a higher biological dose to be administered while shortening the total treatment time [4].

The challenge faced by researchers is the summation of doses from both methods in selected critical organs $[5,6]$. One of the main critical organs in cervical cancer radiotherapy is the bladder.

For EBRT, owing to some tools implemented in treatment planning systems, it is possible to precisely 
measure the parameters of dose distribution in the bladder. In the case of BRT, the applicator-induced artefacts during brachytherapy application substantially reduce the correctness of bladder volume determination and, consequently, limit the assessment of the distribution of deposited doses. During EBRT, the patient is usually in a standard supine position, with a padding placed under her knees. Whereas during BRT, the patient lies in a supine position, with legs spread apart and specially supported to enable a brachytherapy applicator to be inserted. Both patient's position and applicator placement require different morphological conditions than those applied for EBRT [7,8].

To adjust the image of patient's anatomy during BRT (deformed image) to the image of patient's anatomy during EBRT (reference image), rigid image registration allows only the possibility of up-scaling/down-scaling, shifting, and rotating (in each direction of the Cartesian system) of the projected image in relation to the reference image [9]. With deformations of organs (i.e. bladder) not reflected, it is impossible to merge the images, so that anatomical structure visible in the projected image would be appropriately deformed and adapted to the shape in the reference image [7]. Consequently, it is impossible to reconstruct the distribution of doses deposited in particular structures during BRT in images used during the development of EBRT plan. Therefore, a merger of medical images obtained during EBRT and BRT should be based on deformable image registration methods [5].

The main algorithms applied in a deformable image registration are algorithms with diffusive model (demon registration), algorithms using a transformation and analysis of B-spline functions (B-spline registration), and hybrid algorithms, which combine functionalities of both [9]. Deformable registration methods often employ supporting structures, such as triangular meshes generated from contours of selected anatomical structures of patient [10]. The triangular meshes are used to reduce the calculations made during the registration for a selected structure and, thus, reconstructing its deformation more precisely.

It also needs to be remembered that medical images used during BRT and EBRT may be created by means of various imaging methods, e.g. cone-beam computed tomography images (CBCT) often used in BRT planning, and conventional CT images applied for EBRT planning.

The aim of the present study was to assess selected methods of image registration available in the RaySearch treatment planning system (RaySearch Laboratories AB, Stockholm, Sweden) and their impact on the accuracy of mapping of doses deposited in the bladder during BRT in images used during EBRT.

\section{Material and methods}

The study was retrospective in nature. Analysis was made for 10 cervical cancer patients. Each of the patients was treated with BRT using a three-channel Fletcher applicator, and EBRT involving photon beams delivered by a linear accelerator.

\section{Brachytherapy}

Brachytherapy (BRT) dose planning and optimization was based on medical images obtained from Acuity simulator (Varian Medical Systems, Palo Alto, USA). Two orthogonal two-dimensional topographical images (2D-keV) were produced, and CT images were obtained through CBCT. The slice thickness was $0.2 \mathrm{~cm}$, and the pixel size was $0.088 \mathrm{~cm} \times 0.088 \mathrm{~cm}$. All images were made within a single imaging session for a patient set-up used in BRT, with an applicator placed inside the uterine cavity and a catheter placed in the bladder. Based on the $2 \mathrm{D}-\mathrm{keV}$ images, a treatment plan was made for iridium-192 source ( ${ }^{192} \mathrm{Ir}$ ) with radiation energy of $0.397 \mathrm{MeV}$ (treatment planning system BrachyVision version 11; Varian Medical Systems, Palo Alto, USA, algorithm TG-43). Dose distributions were calculated for a homogeneous medium, with density equivalent to that of water. The $2 \mathrm{D}-\mathrm{keV}$ images were merged with the CBCT images using the rigid registration method with a particular focus on bone anatomy compatibility [11]. Then, the planned dose distributions (based on the $2 \mathrm{D}-\mathrm{keV}$ images) were copied onto the CBCT images. The bladder was contoured on the CBCT images.

\section{External beam radiation therapy}

To deliver radiotherapy, each of the patients was CTscanned by Somatom Definition AS scanner (Siemens Medical Solutions, Erlangen, Germany). During CT imaging, the patients were arranged in a supine position with a padding under their knees. They had no applicator placed in the uterus nor catheter in the bladder. The slice thickness was $0.3 \mathrm{~cm}$, and the pixel size was $0.127 \mathrm{~cm} \times 0.127 \mathrm{~cm}$. Structures necessary to create and deliver the EBRT plan, including the bladder, were contoured on CT images. Radiation therapy plans were delivered by photon energy beams of $6 \mathrm{MeV}$ and $15 \mathrm{MeV}$ using IMRT technique (Eclipse version 11 planning system, algorithm AA 11031; Varian Medical Systems, Palo Alto, USA). Total dose of 50.4 Gy was administered in 28 radiation fractions. Bladder contours in both the CBCT and $\mathrm{CT}$ images were made by the same radiotherapist.

\section{Data preparation and migration}

Both the BRT plans created in the BrachyVision planning system and the EBRT plans (Eclipse planning system) were exported to the RaySearch treatment planning system (version 9A), where image registration and dose distribution mapping procedures were carried out. When performing those procedures, the following data were used:

- for BRT plans, dose distribution and CBCT images with bladder contour;

- for EBRT plans, CT images with bladder contour.

To evaluate the correctness of data migration, a comparison was made between volumes of the bladder contoured in Varian systems (BrachyVision, Eclipse) and the same volumes, which were determined based on contours of those structures imported and reconstructed in the RaySearch system. These were: 
- for BRT plan migration (BrachyVision $\rightarrow$ RaySearch), bladder volume calculated on the basis of the contour made in CBCT images;

- for EBRT plan migration (Eclipse $\rightarrow$ RaySearch), bladder volume calculated on the basis of the contour made in CT images.

Protocol of operations performed in the RaySearch system was as follows:

- registration of CT images (from EBRT plan) and CBCT image registration (from BRT plan);

- propagation of the bladder contour based on CBCT images (BRT) and its transfer to CT images (EBRT);

- mapping of dose distributions from the BRT plan in CT images (EBRT), according to the registration result.

\section{CT and CBCT image registration}

CT and CBCT images were registered by three methods: 1 . Rigid registration based on bone structures (Reg_1); 2. Deformable registration using anatomically constrained deformation algorithm (ANACONDA) (Reg_2a and Reg_2b); 3. Deformable registration with biomechanical algorithm (MORFEUS) (Reg_3).

In each case, the CT image set was a reference image, while the CBCT image set was a deformed image. By mapping $\mathrm{CBCT}$ images on $\mathrm{CT}$ images, the bladder contour can be propagated from $\mathrm{CBCT}$ images onto $\mathrm{CT}$ images [12]. However, it should be noted that rigid registration (Reg_1) does not cause the bladder contour to be transferred from CBCT to CT to change its form, but only to change its orientation [13]. Therefore, Reg_1 was used as a starting point for operations, which enabled monitoring of anatomy changes (deformations) between CT and CBCT images.

Accordingly, two deformation algorithms available in the RaySearch system were used. First of them (ANACONDA) is a hybrid algorithm that combines the diffusive model (demon) with that employing the B-spline functions [9]. Registration using that combination can be based on both structure contours and contour-based structure triangular meshes [14]. Both registration models were used in this study: Reg_2a - hybrid registration, where the control area was formed by bladder contours, and Reg_ $2 b$ - hybrid registration, where the control area was formed by a triangular mesh based on the bladder contours. The other algorithm applied was the biomechanical algorithm (MORFEUS) $[15,16]$. Using that algorithm, a registration (Reg_3) was made, where the control area was formed by a triangular mesh based on the bladder contours.

The deformable mesh in all the cases (Reg_2a, Reg_2b, and Reg_3) was $0.3 \mathrm{~cm}$ in the sagittal plane and $0.25 \mathrm{~cm}$ in the frontal and transversal planes.

Sørensen-Dice similarity coefficient (DSC) was used to assess the similarity between the shape of the bladder in the reference image (CT) and that of the bladder from the deformed image (CBCT) transferred to the reference image $[5,17]$ :

$$
D S C=2 \times \frac{V\left(B_{r e f}\right) \cap V\left(B_{d e f}\right)}{V\left(B_{r e f}\right)+V\left(B_{d e f}\right)}
$$

where $\mathrm{V}\left(\mathrm{B}_{\text {ref }}\right)$ is the volume $(\mathrm{V})$ of the bladder $(\mathrm{B})$ defined on the base of contours in the reference image (ref), and
$\mathrm{V}\left(\mathrm{B}_{\mathrm{def}}\right)$ is the volume $(\mathrm{V})$ of the bladder (B) delineated based on contours from the deformed image (def) transferred to the reference image.

DSC assumed values were from 0 (no overlap) to 1 (full overlap).

Furthermore, for methods using a triangular mesh (Reg_1, Reg_2b, and Reg_3), medium and maximum discrepancies were assessed between corresponding points of the reference mesh (in CT images) and deformable mesh (from CBCT images).

\section{Dose distribution mapping}

Dose distributions from the BRT plans were transformed according to registration results, then transferred and associated with data (CT images, bladder contour) representing patients' anatomy during EBRT.

Assuming ideal registration conditions $(\mathrm{DSC}=1)$, dose distribution analysis with BRT in the bladder, visualized in CT images (EBRT), should only show dose displacement in the bladder, while maintaining maximum dose values. However, each non-ideal image registration (DSC < 1) leads to a situation, where deformed bladder contours from CBCT images are not fully aligned with reference contours from CT images. Respecting the above, a comparative analysis was made of BRT doses deposited in $2 \mathrm{~cm}^{3}$ of the bladder, visualized in CBCT images (primary BRT dose distribution) and in CT images (transformed BRT dose distribution).

\section{Statistical analysis}

Wilcoxon test was performed to assess differences between the volume of the bladder determined during assessing treatment plans in the BrachyVision and Eclipse systems, and the volume reconstructed in the RaySearch system. The same test was used to evaluate differences between the volume of the bladder during BRT (visualization in CBCT) and EBRT (visualization in EBRT). Differences between the registration results and dose mapping results were evaluated using Friedman ANOVA test, with supporting post-hoc tests (Nemenyi's procedure). All the tests were performed at a statistical significance level of 0.05 .

\section{Results}

\section{Bladder volume analysis}

The reconstruction of contours in the RaySearch system leads to an increase of bladder volume in relation to that determined in the BrachyVision and Eclipse systems (Table 1). The exception was one observation shown in Table 1 related to the reconstruction of contour in the CT images (EBRT plan) for patient 2 .

The observed increase of the bladder volume was independent on the data source: $\Delta(\mathrm{RS}-\mathrm{BV})$ vs. $\Delta(\mathrm{RS}-\mathrm{E})$; $p=0.557$, respectively. Moreover, there was no correlation found between the volume of the bladder and the extent of its increase caused by the reconstructions performed in the RaySearch system. The coefficients of determination obtained, and the related $p$-values were: $R^{2}=0.011$ 
Table 1. Volumes of the bladder based on the contours made during treatment planning in the BrachyVision and Eclipse systems, and reconstructed after data migration to the RaySearch system

\section{Bladder $\left(\mathrm{cm}^{3}\right)$}

\begin{tabular}{lcccccc}
\hline Patient & \multicolumn{3}{c}{ CBCT images } & \multicolumn{3}{c}{ CT images } \\
\cline { 2 - 7 } & BV & RS & $\Delta$ (RS-BV) & E & RS & $\Delta$ (RS-E) \\
\hline 1 & 56.5 & 57.9 & 1.3 & 91.6 & 92.6 & 1.0 \\
\hline 2 & 87.9 & 88.3 & 0.5 & 209.8 & 209.4 & -0.4 \\
\hline 3 & 127.5 & 128.4 & 1.0 & 34.8 & 35.4 & 0.6 \\
\hline 4 & 111.3 & 112.0 & 0.7 & 124.1 & 124.3 & 0.2 \\
\hline 5 & 46.4 & 46.6 & 0.2 & 354.5 & 355.0 & 0.5 \\
\hline 6 & 37.9 & 38.3 & 0.4 & 56.3 & 56.6 & 0.3 \\
\hline 7 & 61.2 & 62.4 & 1.2 & 51.3 & 52.7 & 1.4 \\
\hline 8 & 74.5 & 75.2 & 0.7 & 215.9 & 218.1 & 2.2 \\
\hline 9 & 48.1 & 48.5 & 0.4 & 70.2 & 71.6 & 1.3 \\
\hline 10 & 203.6 & 204.2 & 0.6 & 236.4 & 237.7 & 1.3
\end{tabular}

$B V$ - BrachyVision, RS-RaySearch, E-Eclipse

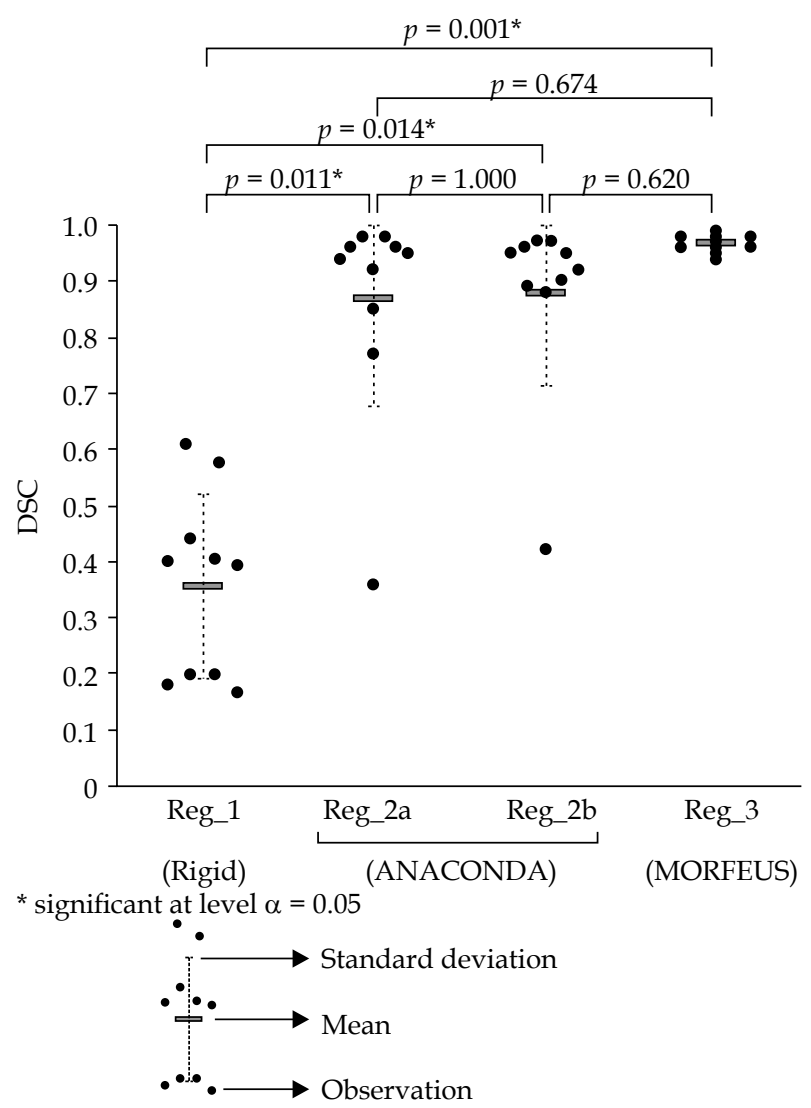

Fig. 2. DSC for bladder obtained for the four methods of registering $\mathrm{CT}$ images from $\mathrm{CBCT}$

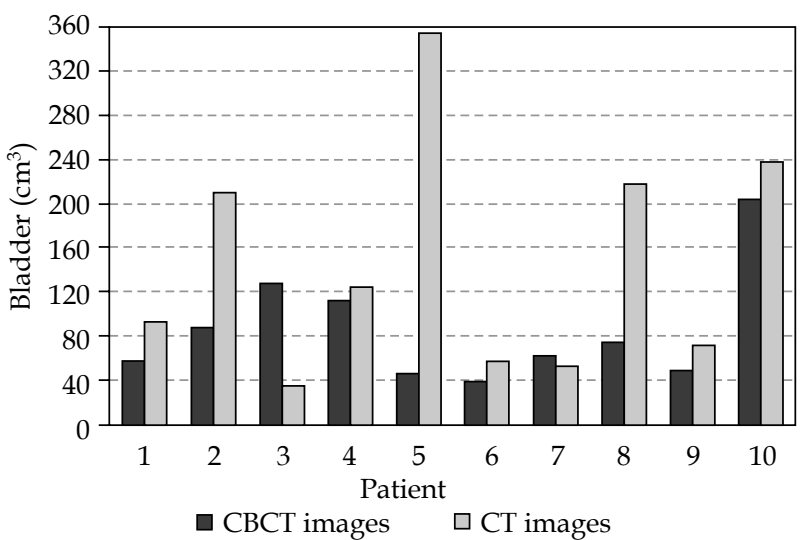

Fig. 1. Bladder volumes during BRT (CBCT images) and during EBRT (CT images)

and $p=0.773$ for BRT (CBCT images) and $R^{2}=0.001$ and $p=0.931$ for EBRT (CT images), respectively.

However, it should be noted that bladder volumes during BRT differed significantly from those observed during EBRT $(p=0.044)$ (Figure 1).

\section{$C T$ and $C B C T$ image registration}

Figure 2 shows DSC values for the bladder obtained using the four different methods of registering CT images from CBCT, i.e.: rigid registration (Reg_1) two hybrid registrations based on bladder contours (Reg_2a) and triangular meshes (Reg_2b), and biomechanical registration (Reg_3). DSC values obtained for deformable registrations were significantly higher than those obtained for rigid registration $(p=0.011$ for Reg_1 vs. Reg_2a; $p=0.014$ for Reg_1 vs. Reg_2b, and $p=0.001$ for Reg_1 vs. Reg_3). No statistically significant differences were found between DSC values for deformable registrations. However, 9 out of 10 observations made for Reg_3 displayed DSC values higher than 0.95 , whereas for hybrid registrations (Reg_2a, Reg_2b), such high DSC values were observed for 4 and 5 observations, respectively (Table 2).

Observations shown in Table 2 were confirmed by analyzing the discrepancies between corresponding triangular mesh points located on the surface of the bladder. The analysis showed a statistically significant reduction of discrepancies for Reg_3 compared to Reg_2b, with $p=0.039$ (Figure 3).

Table 2. Number of observations according to the extent of DSC values for the four registration methods of $C T$ images from $C B C T$

Number of observations

\begin{tabular}{lcccc}
\hline DSC (range) & Rigid & \multicolumn{2}{c}{ ANACONDA } & MORFEUS \\
\cline { 2 - 5 } & Reg_1 & Reg_2a & Reg_2b & Reg_3 \\
\hline$[0.00,0.70]$ & 10 & 1 & 1 & 0 \\
\hline$[0.70,0.90]$ & 0 & 2 & 2 & 0 \\
\hline$[0.90,0.95]$ & 0 & 3 & 2 & 1 \\
\hline$[0.95,1.00]$ & 0 & 4 & 5 & 9
\end{tabular}




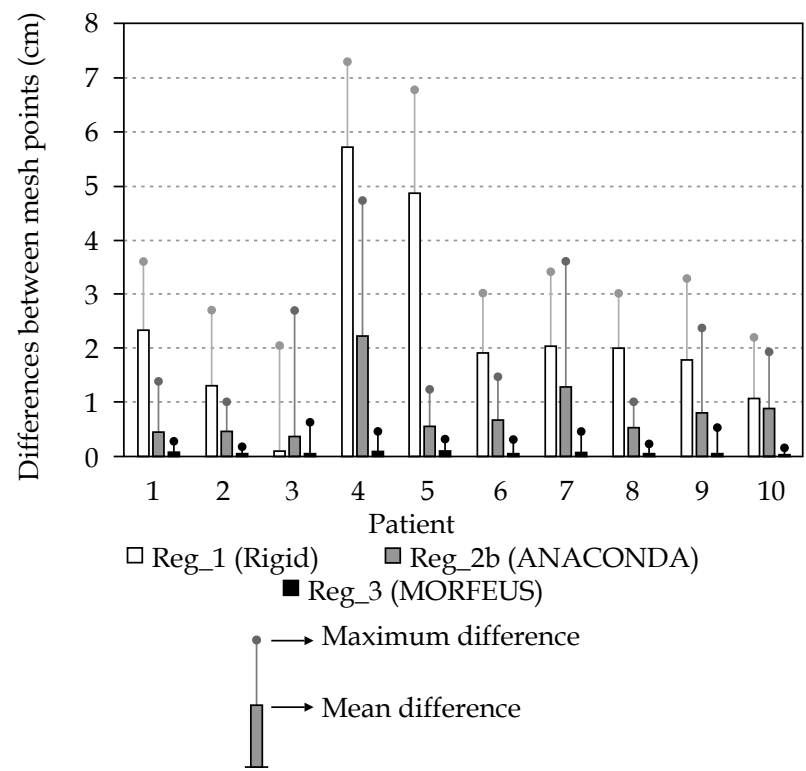

Friedman test, $p$-value $<0.001$

Nemenyi procedure, $p$-values:

\begin{tabular}{|c|c|c|c|}
\hline & Reg_1 & Reg_2b & Reg_3 \\
\hline Reg_1 & 1.000 & 0.175 & $<\mathbf{0 . 0 0 1}$ \\
\hline Reg_2b & & 1.000 & $\mathbf{0 . 0 3 9}$ \\
\hline Reg_3 & & & 1.000 \\
\hline
\end{tabular}

Fig. 3. Differences between corresponding points (from reference and deformed meshes) for three mesh-based registrations

\section{Dose distribution mapping}

Table 3 demonstrates doses deposited in $2 \mathrm{~cm}^{3}$ of the bladder, including doses for the bladder volume determined in CBCT images (initial BRT treatment plan), and doses for BRT distribution transformed by the three deformable registration methods and reading of the bladder volume determined in CT images, when developing the EBRT plan.

Differences between BRT doses in CBCT images in the bladder and the same doses transformed and visualized in CT images are shown in Figure 4.

There are no statistically significant differences between the results for the three registration methods $(p=0.273)$. However, the highest uniformity in all registrations were found in Reg_3 (Figure 4). Moreover, dose mapping in CT images based on Reg_3 had the least error: for Reg_3, 5 out of 10 observations yielded error of less 5\%, while for Reg_2a and Reg_2b, there were only three of such observations. Also, there were some observations where the discrepancy between the original and mapped dose, regardless of the registration method, was unacceptable (data for patients 2, 5, and 8 in Figure 4).

\section{Discussion}

Modern BRT planning systems enable the visualization of doses on CT images by combining information of dose distribution with that of patient's anatomy $[18,19]$.
Table 3. Doses measured for bladder on CBCT images (initial BRT plan) and corresponding doses mapped by three registration methods, and displayed for bladder on CT images

Maximum dose (Gy) measured in $2 \mathrm{~cm}^{3}$ of the bladder

\begin{tabular}{lcccc}
\hline Patient & $\begin{array}{c}\text { CBCT } \\
\text { images }\end{array}$ & \multicolumn{3}{c}{ CT images ${ }^{\circledR}$} \\
\cline { 3 - 5 } & & Reg_2a & Reg_2b & Reg_3 \\
\hline 1 & 5.20 & 5.26 & 5.36 & 5.32 \\
\hline 2 & 9.43 & 10.60 & 11.05 & 11.84 \\
\hline 3 & 6.54 & 6.78 & 7.21 & 7.46 \\
\hline 4 & 3.62 & 3.98 & 3.75 & 3.41 \\
\hline 5 & 9.25 & 19.75 & 12.09 & 12.05 \\
\hline 6 & 4.99 & 4.33 & 4.53 & 4.83 \\
\hline 7 & 2.94 & 2.04 & 2.28 & 2.96 \\
\hline 8 & 5.97 & 7.38 & 7.32 & 6.83 \\
\hline 9 & 3.32 & 3.76 & 3.71 & 3.44 \\
\hline 10 & 7.50 & 7.67 & 7.83 & 7.64 \\
\hline
\end{tabular}

\#BRT doses measured in bladder contoured on CBCT; @BRT doses, mapped on CT and measured in bladder contoured on CT

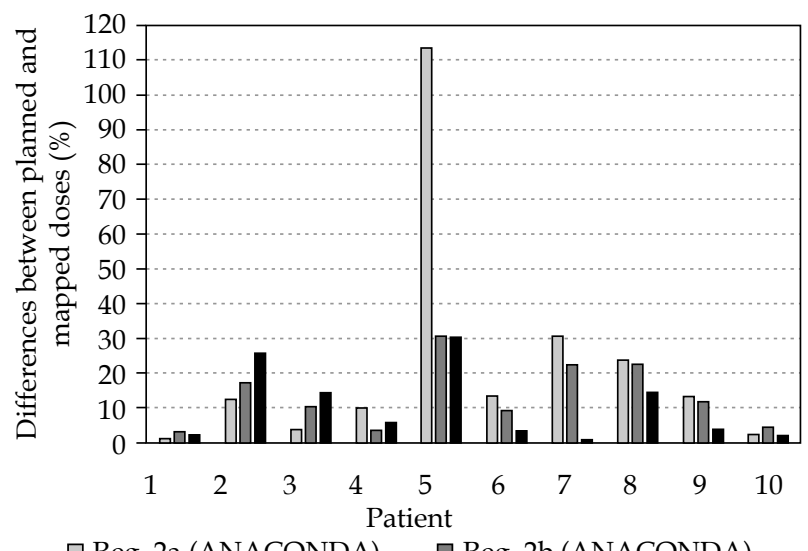

$\square \operatorname{Reg} \_2 a(A N A C O N D A) \quad \square \operatorname{Reg} 2$ 2b (ANACONDA) - Reg_3 (MORFEUS)

Fig. 4. Differences between planned (CBCT) and mapped (CT) doses measured in $2 \mathrm{~cm}^{3}$ of the bladder

The quality of CT images is one of the key factors determining the complexity of dose distribution analysis in patient's body. While CT images enable doses to be measured in most organ/regions during BRT, the quality of CBCT images reduces substantially the extent of those analyses [20]. Figure 5 shows a CBCT image made on an Acuity simulator for a volume treated with BRT. It displays the bladder (contoured with a green line), Foley's catheter placed in the bladder (yellow line), and a three-channel Fletcher applicator (three bright points located centrally in the patient's body). The quality of CBCT images makes it impossible to specify the exact location of the rectum and target volume and, thus, to perform a spatial analysis of doses deposited in those struc- 


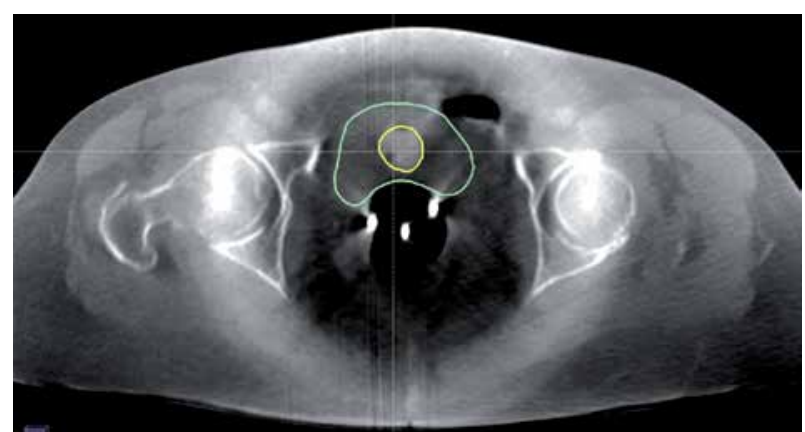

Fig. 5. СBCT image of region treated during brachytherapy

tures. It is, however, possible to analyze dose distribution in the bladder.

The bladder is an organ prone to substantial changes in its volume, shape, and position. As shown in Figure 1,
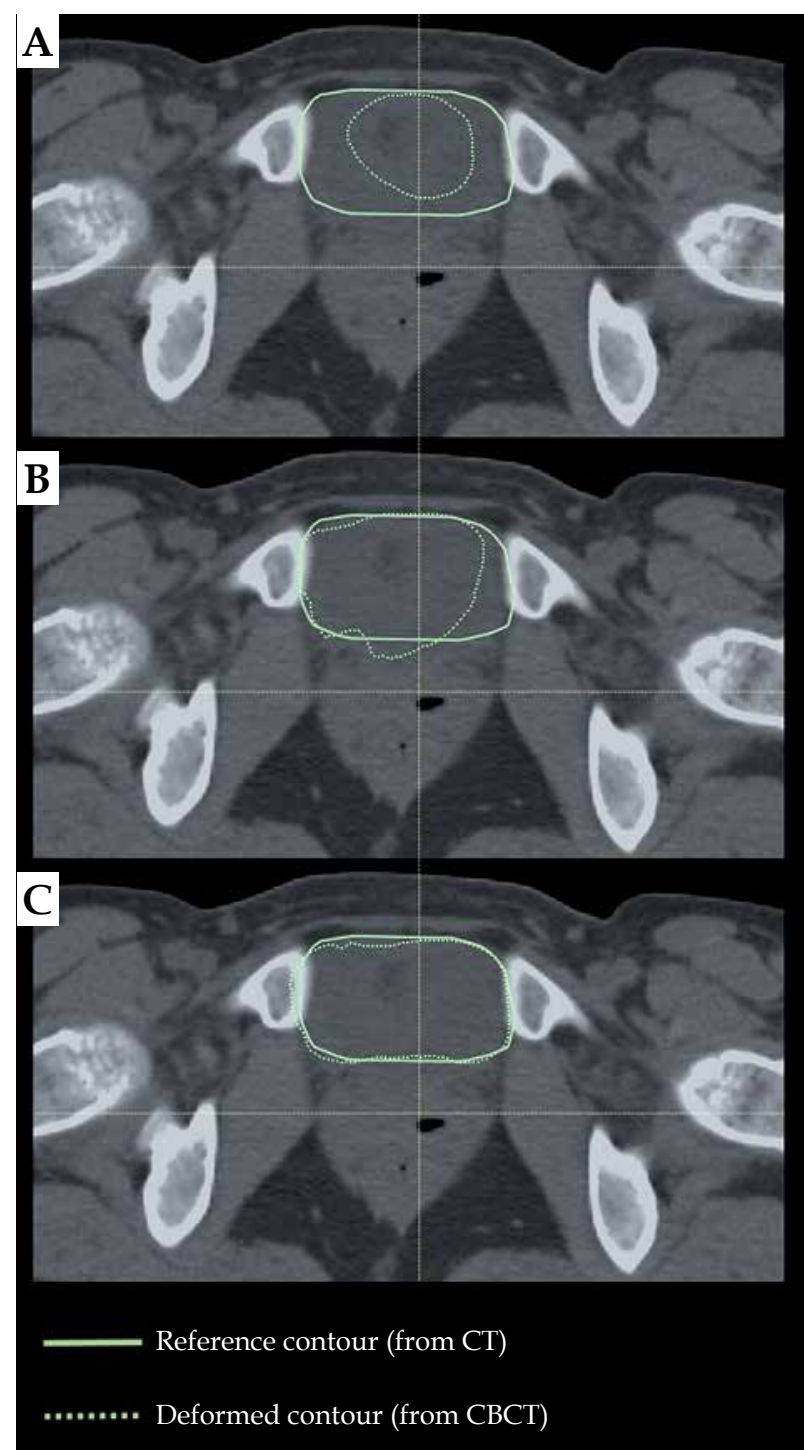

Fig. 6. Alignment of bladder contour from CBCT image (dashed line) with the corresponding reference contour delineated in the CT image (continuous line) for $\mathbf{A}$ ) rigid registration, Reg_1, B) hybrid registration, Reg_2b, and C) biomechanical registration, Reg_3 bladder volume during BRT (CBCT) differs considerably from that during EBRT (CT). Therefore, to map BRT doses in the bladder visualized in CT images (EBRT), advanced image registration methods need to be applied. For the sake of clinical utility, it is important to measure the error caused by those transformations., and for that reason, the present study did not raise issues related to combining doses deposited in particular BRT fractions [21] or combining doses between BRT and EBRT [5]. The study only evaluated the accuracy of image registration and dose-based transformations performed. Reduction of the research to the assessment of accuracy of image registration and dose mapping methods was also dictated by a traditional way of BRT dose calculations, which did not reflect the inhomogeneity of irradiated medium reducing the possibility of combining BRT doses with EBRT doses (calculated in CT images, considering the inhomogeneity of medium) [22].

This study evaluated the methods implemented in the RaySearch system, with data imported from Varian treatment planning systems (BRT - BrachyVision, EBRT - Eclipse). Even though the resolution of images did not change during the import, some differences were observed between the bladder volume (Table 1) measured in the RaySearch system and that measured in the Varian systems. Those differences result from different contour reconstruction methods applied by the BrachyVision/ Eclipse and RaySearch [23,24]. It has to be mentioned that the increase in the bladder volume was the same for CBCT images exported from the BrachyVision as for CT images from the Eclipse (mean growth by $0.76 \%$ ). Therefore, the increase did not distort the evaluations performed by the RaySearch system.

Image registration methods implemented in the RaySearch system enable both rigid and deformable registrations. As shown above, the deformable methods largely improve the quality of image registration as compared to the rigid methods (Figure 2). Evaluating the deformable methods, we demonstrated the biomechanical method to be the most effective one (Reg_3) (Table 2, Figure 3). Figure 6 shows the example alignment of bladder contours based on rigid registration (Reg_1) and deformable reg-

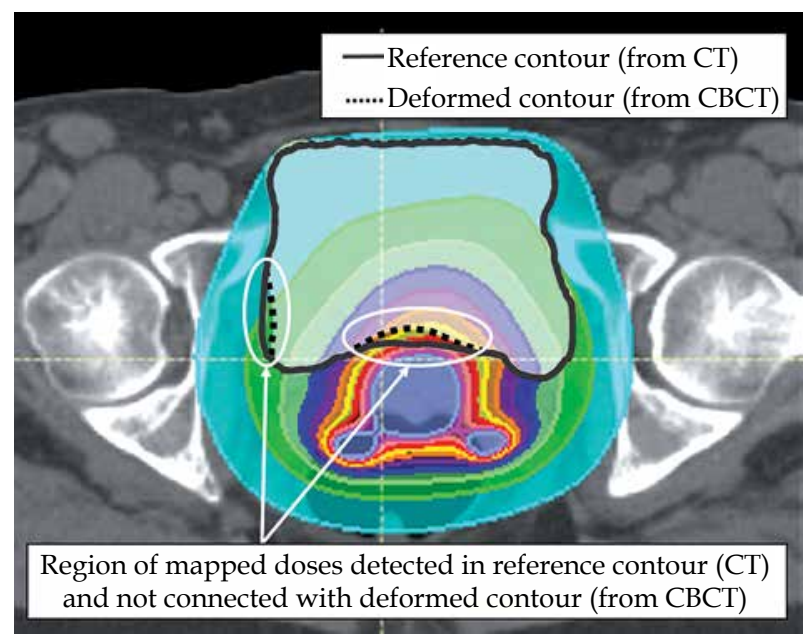

Fig. 7. Example of errors in dose mapping procedure 
istrations: hybrid (Reg_2b) and biomechanical (Reg_3). A triangular mesh based on bladder contours was the control area for all registrations.

BRT doses initially associated with the CBCT image were transformed according to the registration results. Then, they were combined with the CT image (EBRT). Comparing doses in the bladder in CBCT and CT images, the highest compatibility was achieved in the transformation based on the biomechanical registration results (Reg_3). For 5 out of 10 observations, the discrepancy between doses was smaller than $5 \%$ (data patient 1, 6, 7, 9, and 10 in Figure 4). This confirms the observations made by Rigaud et al. [17]. Unfortunately, for 2 out of 10 observations, discrepancies were larger than $15 \%$ (data for patient 2 and 5 in Figure 4). It should be noted that the patients with observed discrepancies were marked with a substantial difference in bladder volume in CBCT and CT images, and relatively low DSC values (0.96) obtained with biomechanical registration (Reg_3). Low DSC values prove the inaccuracy in the alignment of bladder contours in the region where BRT doses are deposited. Therefore, prior to result acceptance, each image registration and contour propagation procedure as well as final dose distribution mappings should be visually evaluated. Figure 7 shows the result of dose mapping obtained for biomechanical registrations (Reg_3), which were characterized by various DSC values.

\section{Conclusions}

Of all registrations analyzed, it is the biomechanical registration (Reg_3) that enables the most accurate alignment between CBCT images used (in this study) during BRT planning, with CT images used in EBRT planning. Satisfactory registration results of anatomical structures do not guarantee a correct mapping of primary BRT doses to the bladder in CT images. In this study, the results of dose transformation based on the biomechanical registration had an error of less than $5 \%$ for only $50 \%$ of the observations.

\section{Disclosure}

The authors report no conflict of interest.

\section{References}

1. Pötter R, Haie-Meder C, Van Limbergen E et al. Recommendations from gynaecological (GYN) GEC ESTRO working group (II): concepts and terms in 3D image-based treatment planning in cervix cancer brachytherapy-3D dose volume parameters and aspects of 3D image-based anatomy, radiation physics, radiobiology. Radiother Oncol 2006; 78: 67-77.

2. Ito $H$, Kutuki S, Nishiguchi I et al. Radiotherapy for cervical cancer with high-dose rate brachytherapy-correlation between tumor size, dose and failure. Radiother Oncol 1994; 31: 240-247.

3. Kirisits C, Pötter R, Lang $S$ et al. Dose and volume parameters for MRI-based treatment planning in intracavitary brachytherapy for cervical cancer. Int J Radiat Oncol Biol Phys 2005; 62: 901-911.

4. De Leeuw AA, Van de Kamer JB, Moerland MA et al. The effect of alternative biological modelling parameters $(\alpha / \beta$ and half time of repair T1/2) on reported EQD2 values in the treatment of advanced cervical cancer. Radiother Oncol 2011; 101: 337-342.

5. Andersen ES, Noe KØ, Sørensen TS et al. Simple DVH parameter addition as compared to deformable registration for bladder dose accumulation in cervix cancer brachytherapy. Radiother Oncol 2013; 107: 52-57.

6. Kadoya N, Miyasaka Y, Nakajima Y et al. Evaluation of deformable image registration between external beam radiotherapy and HDR brachytherapy for cervical cancer with a 3D-printed deformable pelvis phantom. Med Phys 2017; 44: 1445-1455.

7. Jadon R, Pembroke CA, Hanna CL et al. A systematic review of organ motion and image-guided strategies in external beam radiotherapy for cervical cancer. Clin Oncol 2014; 26: 185-196.

8. Kikuchi K, Nakamura R, Tanji S et al. Three-dimensional summation of rectal doses in brachytherapy combined with external beam radiotherapy for prostate cancer. Radiother Oncol 2013; 107: 159-164.

9. Czajkowski P, Piotrowski T. Registration methods in radiotherapy. Rep Pract Oncol Radiother 2019; 24: 28-34.

10. Kaus MR, Brock KK, Pekar V et al. Assessment of a model-based deformable image registration approach for radiation therapy planning. Int J Rad Oncol Biol Phys 2007; 68: 572-580.

11. Sabater S, Andres I, Sevillano M et al. Dose accumulation during vaginal cuff brachytherapy based on rigid/deformable registration vs. single plan addition. Brachytherapy 2014; 13: 343-351.

12. Flower E, Do V, Sykes J et al. Deformable image registration for cervical cancer brachytherapy dose accumulation: organ at risk dose-volume histogram parameter reproducibility and anatomic position stability. Brachytherapy 2017; 16: 387-392.

13. Ryckman JM, Shelton JW, Waller AF et al. Anatomic structure-based deformable image registration of brachytherapy implants in the treatment of locally advanced cervix cancer. Brachytherapy 2016; 15: 584-592.

14. Weistrand O, Svensson $\mathrm{S}$. The ANACONDA algorithm for deformable image registration in radiotherapy. Med Phys 2015; 42: 40-53.

15. Brock KK, Sharpe MB, Dawson LA et al. Accuracy of finite element model-based multi-organ deformable image registration. Med Phys 2005; 32: 1647-1659.

16. Velec M, Moseley JL, Svensson S et al. Evaluation of biomechanical deformable image registration (DIR) in a commercial radiation therapy planning system. Int J Rad Oncol Biol Phys 2015; 93: S215-S216.

17. Rigaud B, Klopp A, Vedam S et al. Deformable image registration for dose mapping between external beam radiotherapy and brachytherapy images of cervical cancer. Phys Med Biol 2019; 64: 115023.

18. van Heerden LE, Houweling AC, Koedooder K et al. Structure-based deformable image registration: added value for dose accumulation of external beam radiotherapy and brachytherapy in cervical cancer. Radiother Oncol 2017; 123: 319-324.

19. Vásquez Osorio EM, Kolkman-Deurloo IK, Schuring-Pereira $\mathrm{M}$ et al. Improving anatomical mapping of complexly deformed anatomy for external beam radiotherapy and brachytherapy dose accumulation in cervical cancer. Med Phys 2015; 42: 206-220.

20. Abe T, Tamaki T, Makino S et al. Assessing cumulative dose distributions in combined radiotherapy for cervical cancer using deformable image registration with pre-imaging preparations. Radiat Oncol 2014; 9: 293.

21. Mohammadi R, Mahdavi S, Jaberi R et al. Evaluation of deformable image registration algorithm for determination of 
accumulated dose for brachytherapy of cervical cancer patients. J Contemp Brachytherapy 2019; 11: 469-478.

22. Jamema SV, Mahantshetty U, Andersen E et al. Uncertainties of deformable image registration for dose accumulation of high-dose regions in bladder and rectum in locally advanced cervical cancer. Brachytherapy 2015; 14: 953-962.

23. RayStation 9A, user manual, Workspace Main version a487, checked in 2019-06-20, Skribenta version 5.3.027.

24. Varian, Eclipse Algorithms Reference Guide Eclipse, P/N B503486R01B, December 2011. 